IRSTI 27.29 .23

\author{
${ }^{1}$ B. Bekbolat, ${ }^{2}$ N. Tokmagambetov \\ ${ }^{1}$ Al-Farabi Kazakh National University, Almaty, Kazakhstan \\ ${ }^{2}$ Institute of Mathematics and Mathematical Modeling, Almaty, Kazakhstan \\ e-mail: tokmagambetov@math.kz

\section{On a boundedness result of non-toroidal pseudo-differential operators}

\begin{abstract}
In this article, we prove boundedness results for $\theta$-toroidal pseudo-differential operators generated by a differentiation operator with a non-periodic boundary condition. $\theta$-toroidal pseudodifferential operators are a natural generalization of a toroidal one. As in the classical case, this class of operators act on a suitable test function space by weighting the Fourier transform "very well". Standard operations as adjoints, products and commutators with $\theta$-toroidal pseudo-differential operators can be characterized by their $\theta$-toroidal symbols. For pseudo-differential operators on $\mathrm{R}^{\mathrm{n}}$, the symbol analysis is well developed. Here, we provide more complicated properties of the $\theta$-toroidal pseudo calculus. Namely, we introduce a Holder space induced by a differentiation operator with a non-periodic boundary condition. Finally, for the elements of this space we prove theorems on boundedness of the operators acting on the specified functional spaces. Indeed, in this paper we continue a development of the so called "nonharmonic analysis" introduced in the recent papers of the authors.

Key words: $\theta$-toroidal pseudo-differential operator, $\theta$-toroidal Holder space, $\theta$-Fourier transform, $\theta$ symbol, bounded operator.
\end{abstract}

\section{Introduction}

In [3], it was introduced an analysis generated by the differential operator

$$
\operatorname{Ly}(x)=-i \frac{d y(x)}{d x}, 0<x<1
$$

acting on $\mathrm{L}_{2}(0 ; 1)$ with the boundary condition

$$
\theta y(0)-y(1)=0,
$$

where $\theta \geq 1$.

Spectrum of the operator $L$ is

$$
\lambda_{\xi}=-i \ln \theta+2 \xi \pi, \quad \xi \in \mathrm{Z}
$$

System of eigenfunctions of the operator $L$ is

$$
u_{\xi}(x)=\theta^{x} e^{i 2 \xi \pi x}, \xi \in Z .
$$

and the biorthogonal system to $\mathrm{u}_{\xi}(\mathrm{x})$ in $\mathrm{L}_{2}(0 ; 1)$ is

$$
\mathrm{v}_{\xi}(\mathrm{x})=\theta^{-\mathrm{x}} \mathrm{e}^{\mathrm{i} 2 \xi \pi \mathrm{x}}, \xi \in \mathrm{Z} \text {. }
$$

For the following spectral properties of the operator L we refer to [3] and [7].

1. The system of eigenfunctions of the operator $\mathrm{L}$ is a Riesz basis in $\mathrm{L}_{2}(0 ; 1)$;

2. If function $\mathrm{f}$ belongs to the domain of operator $\mathrm{L}$, then $\mathrm{f}(\mathrm{x})$ expands to a uniformly convergent series of eigenfunctions of the operator $\mathrm{L}$;

3 . The resolvent of the operator $\mathrm{L}$ is

$$
(\mathrm{L}-\lambda \mathrm{I})^{-1} \mathrm{f}(\mathrm{x})=
$$

$=i \frac{e^{i \lambda(x+1)}}{\Delta(\lambda)} \int_{0}^{1} e^{-i \lambda t} f(t) d t+i e^{i \lambda x} \int_{0}^{x} e^{-i \lambda t} f(t) d t$, 
Where $\Delta(\lambda)=\theta-\mathrm{e}^{\mathrm{i} \lambda}$.

\section{$\theta$-toroidal pseudo-differential operators}

$\theta$-Fourier transform and $\theta$-toroidal Hölder spaces. Here we give a definition of $\theta$-Fourier transform [3]. $\theta$-Fourier transform $\quad(\mathrm{f} \mapsto$ $\hat{\mathrm{f}}): \mathrm{C}_{\theta}^{\infty}[0,1] \rightarrow \mathrm{S}(\mathrm{Z})$ is given by

$$
\hat{f}(\xi):=\int_{0}^{1} f(x) \overline{v_{\xi}(x)} d x
$$

and its inverse $\hat{f}(\xi)^{-1}$ is given by

$$
f(x)=\sum_{\xi \in Z} \hat{f}(\xi) u_{\xi}(x) .
$$

Remark 1 The functional space $\mathrm{C}_{\theta}^{\infty}[0,1]$ is called the space of test functions and $\mathrm{S}(\mathrm{Z})$ is space of rapidly decaying functions [3].

In what follows, we will use the following spaces from [5].

We define $\theta$-toroidal Hölder spaces

$$
\begin{gathered}
\Lambda^{\mathrm{s}}([0,1], \theta)= \\
=\left\{\mathrm{f}:[0,1] \rightarrow \mathrm{C}:|\mathrm{f}|_{\Lambda^{\mathrm{s}}}=\sup _{\mathrm{x}, \mathrm{h} \in[0,1]} \frac{|\mathrm{f}(\mathrm{x}+\mathrm{h})-\mathrm{f}(\mathrm{x})|}{|\mathrm{h}|^{\mathrm{s}}}<\infty\right\} \\
\Lambda_{0}^{\mathrm{s}}([0,1], \theta)=\left\{\mathrm{f} \in \Lambda^{\mathrm{s}}([0,1], \theta): \mathrm{f}(0)=0\right\}
\end{gathered}
$$

for each $0<s \leq 1$. These spaces are Banach.

$\theta$-toroidal symbol class. Suppose that $\mathrm{m} \in$ $\mathrm{R}, 0 \leq \delta, \rho \leq 1$. Then the $\theta$-toroidal symbol class $S_{\delta, \rho}^{m}([0,1] \times Z)$ consists of those function $a(x, \xi)$ which are smooth in $\mathrm{x}$ for all $\xi \in \mathrm{Z}$, and which satisfy

$$
\left|\Delta_{\xi}^{\alpha} \partial_{\mathrm{x}}^{\beta} \mathrm{a}(\mathrm{x}, \xi)\right| \leq \mathrm{C}_{\mathrm{a} \alpha \beta \mathrm{m}}\langle\xi\rangle^{\mathrm{m}-\rho \alpha+\delta \beta}
$$

for every $\xi \in \mathrm{Z}, \mathrm{x} \in[0,1], \alpha, \beta \in \mathrm{Z}_{+}$, where

$$
\langle\xi\rangle:=1+|\xi| \text {. }
$$

We call $\mathrm{a}(\mathrm{x}, \xi)$ a symbol [3]. The operator $\Delta_{\xi}$ is the difference operator

$$
\Delta_{\xi} \widehat{\sigma}(\xi):=\widehat{\mathrm{e} \sigma}(\xi),
$$

where $\widehat{\sigma}(\xi): Z \rightarrow C$.

We denote the $\theta$-toroidal pseudo-differential operator by

$$
\mathrm{a}(X, D) f(x)=\sum_{\xi \in Z} u_{\xi}(x) a(x, \xi) \hat{f}(\xi)
$$

where $a(x, \xi)$ is a symbol of a $\theta$-toroidal pseudodifferential operator [5].

We can write for $h \in T$,

$$
\begin{gathered}
a(X, D) f(x+h)= \\
=\sum_{\xi \in Z} u_{\xi}(x) a(x+h, \xi) \int_{0}^{1} f(y+h) \overline{v_{\xi}(x)} d y .
\end{gathered}
$$

Theorem 1. (Bernstein). Assume that $f \in$ $\Lambda_{0}^{\mathrm{s}}([0,1], \theta)$, for $\mathrm{s}>\frac{1}{2}$. Then we have $|\hat{\mathrm{f}}|_{\mathrm{L}^{1}(\mathrm{Z})} \leq$ $\mathrm{C}_{\mathrm{s}}\|\mathrm{f}\|_{\Lambda^{s}}$.

Proof. We prove this statement by recalling a definition of the norm

$$
\begin{gathered}
|\hat{\mathrm{f}}|_{\mathrm{L}^{1}(\mathrm{Z})}=\sum_{\xi \in Z}|\hat{\mathrm{f}}(\xi)|=\sum_{\xi \in Z}\left|\int_{0}^{1} \mathrm{f}(\mathrm{x}) \overline{\mathrm{v}_{\xi}(\mathrm{x})} \mathrm{dx}\right| \leq \sum_{\xi \in \mathrm{Z}} \int_{0}^{1}|\mathrm{f}(\mathrm{x})| \mathrm{dx}= \\
=\sum_{\xi \in \mathrm{Z}} \int_{0}^{1} \frac{|\mathrm{f}(\mathrm{x})-\mathrm{f}(0)|}{|\mathrm{x}|^{\mathrm{s}}}|\mathrm{x}|^{\mathrm{s}} \mathrm{dx} \leq \sum_{\xi \in \mathrm{Z}} \int_{0}^{1} \sup _{\mathrm{x} \in[0,1]} \frac{|\mathrm{f}(\mathrm{x})-\mathrm{f}(0)|}{|\mathrm{x}|^{\mathrm{s}}}|\mathrm{x}|^{\mathrm{s}} \mathrm{dx} \\
\leq \sum_{\xi \in \mathrm{Z}}|\mathrm{f}|_{\Lambda^{s}} \int_{0}^{1} \mathrm{x}^{\mathrm{s}} \mathrm{dx} \leq \sum_{\xi \in \mathrm{Z}} \frac{1}{\mathrm{~s}+1}|\mathrm{f}|_{\Lambda^{s}} \leq \sum_{\xi \in \mathrm{Z}} \frac{1}{\mathrm{~s}+1}\left(|\mathrm{f}|_{\Lambda^{s}}+\sup _{\mathrm{x} \in[0,1]}|\mathrm{f}(\mathrm{x})|\right)=\left(\sum_{\xi \in Z} \frac{1}{\mathrm{~s}+1}\right)\|\mathrm{f}\|_{\Lambda^{s}} \leq \mathrm{C}_{\mathrm{s}}\|\mathrm{f}\|_{\Lambda^{s}} .
\end{gathered}
$$


Finally, we proved the theorem.

\section{Boundedness for $\theta$-toroidal pseudo- differential operator}

Here we prove similiar theorems as in [5].

Theorem 2. Let $a(X, D)=a(D)$ be a pseudodifferential operator with symbol $a(\xi)$ depending only on the discrete variable $\xi$. If $\mathrm{a}(\xi) \in \mathrm{L}^{1}(\mathrm{Z})$, then

$$
|\mathrm{a}(\mathrm{D}) \mathrm{f}|_{\Lambda^{\mathrm{s}}} \leq|\mathrm{a}|_{\mathrm{L}^{1}(\mathrm{Z})}|\mathrm{f}|_{\Lambda^{\mathrm{s}}}
$$

for $0<s \leq 1$

Proof. By the formula, we have

$$
\begin{gathered}
a(X, D) f(x+h)-a(X, D) f(x)= \\
=\sum_{\xi \in Z} u_{\xi}(x) a(\xi) \int_{0}^{1}(f(y+h)-f(y)) \overline{v_{\xi}(y)} d y .
\end{gathered}
$$

Thus, we obtain

$$
\begin{gathered}
\frac{|\mathrm{a}(\mathrm{X}, \mathrm{D}) \mathrm{f}(\mathrm{x}+\mathrm{h})-\mathrm{a}(\mathrm{X}, \mathrm{D}) \mathrm{f}(\mathrm{x})|}{|\mathrm{h}|^{\mathrm{s}}} \leq \\
\leq \sum_{\xi \in \mathrm{Z}}\left(|\mathrm{a}(\xi)| \int_{0}^{1} \frac{|\mathrm{f}(\mathrm{y}+\mathrm{h})-\mathrm{f}(\mathrm{y})|}{|\mathrm{h}|^{\mathrm{s}}} \mathrm{dy}\right) . \\
\frac{|\mathrm{a}(\mathrm{X}, \mathrm{D}) \mathrm{f}(\mathrm{x}+\mathrm{h})-\mathrm{a}(\mathrm{X}, \mathrm{D}) \mathrm{f}(\mathrm{x})|}{\left.|\mathrm{h}|^{\mathrm{s}}\left(\mathrm{x}_{\mathrm{h}}, \xi\right)\right) .} \leq \sum_{\xi \in \mathrm{Z}}|\mathrm{h}|^{1-\mathrm{s}}\left|\mathrm{a}\left(\mathrm{x}_{\mathrm{h}}, \xi\right) \ln \theta+\mathrm{i} 2 \pi \xi \mathrm{a}\left(\mathrm{x}_{\mathrm{h}}, \xi\right)+\mathrm{a}^{\prime}\left(\mathrm{x}_{\mathrm{h}}, \xi\right)\right||\hat{\mathrm{f}}(\xi)| \leq \\
\leq \sum_{\xi \in Z} \mathrm{C}_{1}\left(|\ln \theta|\left|\mathrm{a}\left(\mathrm{x}_{\mathrm{h}}, \xi\right)\right|+2 \pi|\xi|\left|\mathrm{a}\left(\mathrm{x}_{\mathrm{h}}, \xi\right)\right|+\left|\mathrm{a}^{\prime}\left(\mathrm{x}_{\mathrm{h}}, \xi\right)\right|\right)|\hat{\mathrm{f}}(\xi)| \leq \sum_{\xi \in \mathrm{Z}} \mathrm{C}_{1}\left(\mathrm{C}\langle\xi\rangle^{\mathrm{m}}+|\xi| \mathrm{C}\langle\xi\rangle^{\mathrm{m}}+\mathrm{C}\langle\xi\rangle^{\mathrm{m}+\delta}\right)|\hat{\mathrm{f}}(\xi)| \leq \\
\leq \sum_{\xi \in Z} \mathrm{C}_{1}\left(\mathrm{C}\langle\xi\rangle^{\mathrm{m}}+\mathrm{C}\langle\xi\rangle^{\mathrm{m}+1}+\mathrm{C}\langle\xi\rangle^{\mathrm{m}+\delta}\right)|\hat{\mathrm{f}}(\xi)| \leq \sum_{\xi \in \mathrm{Z}} 3 \mathrm{CC}_{1}|\hat{\mathrm{f}}(\xi)| \leq 3 \mathrm{CC}_{1} \mathrm{C}_{\mathrm{s}}\|\mathrm{f}\|_{\Lambda^{s} .}
\end{gathered}
$$

Thus,

$$
|\mathrm{a}(\mathrm{X}, \mathrm{D}) \mathrm{f}|_{\Lambda^{\mathrm{s}}} \leq \mathrm{M}\|\mathrm{f}\|_{\Lambda^{\mathrm{s}}}
$$

The next theorem gives a single sufficient condition on the symbol $a(x, \xi)$ for the corresponding pseudo-differential operator

$$
\mathrm{a}(\mathrm{X}, \mathrm{D}): \Lambda_{0}^{\mathrm{s}}([0,1], \theta) \rightarrow \Lambda^{\mathrm{s}}([0,1], \theta)
$$

Finally, we get

$$
|\mathrm{a}(\mathrm{D}) \mathrm{f}|_{\Lambda^{s}} \leq\left(\sum_{\xi \in \mathrm{Z}}|\mathrm{a}(\xi)|\right)|\mathrm{f}|_{\Lambda^{s}}
$$

Theorem 3. Let $\frac{1}{2}<s<1$ and a $\in$ $\mathrm{S}_{\delta, \rho}^{-\mathrm{m}}([0,1] \times \mathrm{Z}), \mathrm{m} \geq 1$. Then there exists $\mathrm{M}>0$ such that

$$
|\mathrm{a}(\mathrm{X}, \mathrm{D}) \mathrm{f}|_{\Lambda^{\mathrm{s}}} \leq \mathrm{M}\|\mathrm{f}\|_{\Lambda^{\mathrm{s}}}
$$

Proof. By the mean value theorem, there exists $\mathrm{x}_{\mathrm{h}} \in[0,1]$ such that

$$
\begin{gathered}
u_{\xi}(x+h) a(x+h, \xi)-u_{\xi}(x) a(x, \xi)= \\
=h u_{\xi}\left(x_{h}\right)\left(a\left(x_{h}, \xi\right) \ln \theta+i 2 \pi \xi a\left(x_{h}, \xi\right)+\right. \\
\left.+a^{\prime}\left(x_{h}, \xi\right)\right) .
\end{gathered}
$$




$$
\begin{gathered}
a(X, D) f(x+h)-a(X, D) f(x)= \\
=\sum_{\xi \in Z} u_{\xi}(x)\left(a(x+h, \xi) \int_{0}^{1} f(y+h) \overline{v_{\xi}(y)} d y-a(x, \xi) \int_{0}^{1} f(y) \overline{v_{\xi}(y)} d y\right)= \\
=\sum_{\xi \in Z} u_{\xi}(x)\left(a(x+h, \xi) \int_{0}^{1}(f(y+h)-f(y)) \overline{v_{\xi}(y)} d y\right)+ \\
+\sum_{\xi \in Z} u_{\xi}(x)\left((a(x+h, \xi)-a(x, \xi)) \int_{0}^{1} f(y) \overline{v_{\xi}(y)} d y\right) .
\end{gathered}
$$

Therefore, using the value mean theorem, we obtain

$$
\begin{aligned}
& \frac{|a(X, D) f(x+h)-a(X, D) f(x)|}{|h|^{s}} \\
& \leq \sum_{\xi \in Z}\left(|a(x+h, \xi)| \int_{0}^{1} \frac{|f(y+h)-f(y)|}{|h|^{s}} d y+\frac{|a(x+h, \xi)-a(x, \xi)|}{|h|^{s}} \int_{0}^{1}|f(y)| d y\right) \\
& \leq \sum_{\xi \in Z}\left(|a(x+h, \xi)| \int_{0}^{1} \frac{|f(y+h)-f(y)|}{|h|^{s}} d y+\frac{|h|\left|a^{\prime}\left(x_{h}, \xi\right)\right|}{|h|^{s}} \int_{0}^{1}|f(y)| d y\right) \\
& \leq \sum_{\xi \in Z}\left(\mathrm{C}\langle\xi\rangle^{-\mathrm{m}}|\mathrm{f}|_{\Lambda_{0}^{s}}+|\mathrm{h}|^{1-\mathrm{s}} \mathrm{C}\langle\xi\rangle^{-\mathrm{m}+\delta} \int_{0}^{1}|\mathrm{f}(\mathrm{y})| \mathrm{dy}\right) \leq \sum_{\xi \in \mathrm{Z}}\left(\mathrm{C}\langle\xi\rangle^{-\mathrm{m}}|\mathrm{f}|_{\Lambda_{0}^{s}}+|\mathrm{h}|^{1-\mathrm{s}} \mathrm{C} \int_{0}^{1} \frac{|\mathrm{f}(\mathrm{y})-\mathrm{f}(0)|}{|y|^{\mathrm{s}}}|\mathrm{y}|^{\mathrm{s}} \mathrm{dy}\right) \\
& \leq|\mathrm{f}|_{\Lambda_{0}^{\mathrm{s}}}\left(\sum_{\xi \in \mathrm{Z}} \mathrm{C}\langle\xi\rangle^{-\mathrm{m}+\delta}\right)\left(\langle\xi\rangle^{-\delta}+\frac{1}{\mathrm{~s}+1}\right) \leq|\mathrm{f}|_{\Lambda_{0}^{\mathrm{s}}}\left(\sum_{\xi \in \mathrm{Z}} \mathrm{C}\langle\xi\rangle^{-\mathrm{m}+\delta}\right)\left(1+\frac{1}{\mathrm{~s}+1}\right) .
\end{aligned}
$$

Finally, we obtain

$$
|\mathrm{a}(\mathrm{X}, \mathrm{D}) \mathrm{f}|_{\Lambda^{\mathrm{s}}} \leq|\mathrm{f}|_{\Lambda_{0}^{s}}\left(\sum_{\xi \in \mathrm{Z}} \mathrm{C}\langle\xi\rangle^{-\mathrm{m}+\delta}\right)\left(1+\frac{1}{\mathrm{~s}+1}\right)
$$

Remark. It follows from the proof of Theorem 4 that

$$
|\mathrm{a}(\mathrm{X}, \mathrm{D}) \mathrm{f}|_{\Lambda^{\mathrm{s}}} \leq|\mathrm{f}|_{\Lambda_{0}^{\mathrm{s}}} \sum_{\xi \in \mathrm{Z}}\left(\mathrm{C}\langle\xi\rangle^{-\mathrm{m}+\delta}+\frac{|\mathrm{a}(\cdot, \xi)|_{\Lambda_{0}^{\mathrm{s}}}}{\mathrm{s}+1}\right) .
$$

So, if $|\mathrm{a}(\cdot, \xi)|_{\Lambda_{0}^{s}} \in \mathrm{L}^{1}(\mathrm{Z})$, then $|\mathrm{a}(\mathrm{X}, \mathrm{D}) \mathrm{f}|_{\Lambda^{s}} \leq$ $\mathrm{M}|\mathrm{f}|_{\Lambda_{0}^{s}}$, with $\mathrm{M}=\sum_{\xi \in \mathrm{Z}} \mathrm{C}_{1}\left(\mathrm{C}\langle\xi\rangle^{-\mathrm{m}+\delta}+\frac{|\mathrm{p}(\cdot, \xi)|_{\Lambda_{0}^{\mathrm{s}}}}{\mathrm{s}+1}\right)<$ $\infty$. In conclusion, the operator $\mathrm{a}(\mathrm{X}, \mathrm{D})$ will be bounded from $\Lambda_{0}^{s}([0,1], \theta)$ into $\Lambda^{s}([0,1], \theta)$. So we obtain the next result:

Theorem 5. Let $s \neq 1, \mathrm{~m}>1$ and $|\mathrm{a}(\cdot, \xi)|_{\Lambda_{0}^{s}} \in$ $\mathrm{L}^{1}(\mathrm{Z})$. If $\mathrm{a} \in \mathrm{S}_{\delta, \rho}^{-\mathrm{m}}$ then $\mathrm{a}(\mathrm{X}, \mathrm{D}): \Lambda_{0}^{\mathrm{s}}([0,1], \theta) \rightarrow$ $\Lambda^{\mathrm{S}}([0,1], \theta)$ is a bounded operator. 
Theorem 6. Let $0<\varepsilon<1$ and $\mathrm{k} \in \mathrm{N}$ with $\mathrm{k}>$ $\frac{\mathrm{n}}{2}$, let a be a symbol such that $\left|\Delta_{\xi}^{\alpha} \mathrm{a}(\mathrm{x}, \xi)\right| \leq$ $\mathrm{C}_{\alpha}\langle\xi\rangle^{-\frac{\mathrm{n}}{2} \varepsilon-(1-\varepsilon)|\alpha|},\left|\partial_{\mathrm{x}}^{\beta} \xi \mathrm{a}(\mathrm{x}, \xi)\right| \leq \mathrm{C}_{\beta}\langle\xi\rangle^{-\frac{\mathrm{n}}{2}}$, for $|\alpha|,|\beta| \leq \mathrm{k}$. Then, $\mathrm{a}(\mathrm{X}, \mathrm{D})$ is a bounded operator from $\mathrm{L}^{\mathrm{p}}(\mathrm{T})$ into $\mathrm{L}^{\mathrm{p}}(\mathrm{T})$ for $2 \leq \mathrm{p}<\infty$.

In the following theorems, we obtain Hölder boundedness using the Morrey inequality [6]: if $1<$ $p<\infty$ and $\mathrm{s}_{\mathrm{p}}=1-\frac{1}{\mathrm{p}}$, then for $\mathrm{x}, \mathrm{y} \in \mathrm{R}$ we have

$$
\frac{|u(x+h)-u(x)|}{|h|^{S_{p}}} \leq\left|u^{\prime}(x)\right|_{L^{p}(R)}
$$

Function on the torus may be thought as those functions on $\mathrm{R}$ that are 1-periodic, under these assumptions, we can use a toroidal version of Morrey inequality on $\mathrm{L}^{\mathrm{p}}(\mathrm{T})$.

Theorem 7. Let $0 \leq \delta<\rho \leq 1$ and $m>1$. If $\mathrm{a} \in \mathrm{S}_{\delta, \rho}^{-\mathrm{m}}$, then $\mathrm{a}(\mathrm{X}, \mathrm{D}): \Lambda_{0}^{\frac{1}{2}}([0,1], \theta) \rightarrow \Lambda^{\frac{1}{2}}([0,1], \theta)$ is a bounded operator.

Proof. The composition of the pseudodifferential operators $\frac{d}{d x}$ and $a(X, D)$ is the pseudodifferential operator $\frac{d}{d x} a(X, D)$ of degree $-m+1<$ 0 , so, $T=\frac{d}{d x} a(X, D): L^{2}(0,1) \rightarrow L^{2}(0,1)$. If $u \in$ $\Lambda_{0}^{\frac{1}{2}}([0,1], \theta)$, then

$$
\begin{aligned}
& \frac{|a(X, D) f(x+h)-a(X, D) f(x)|}{|h|^{S p}} \leq C\left|\frac{d}{d x} a(X, D) u\right|_{L^{2}} \leq C\left\|\frac{d}{d x} a(X, D)\right\|_{\left(L^{2}, L^{2}\right)}|u|_{L^{2}} \\
= & C\left\|\frac{d}{d x} a(X, D)\right\|_{\left(L^{2}, L^{2}\right)}\left(\int_{0}^{1} \frac{|u(x)-u(0)|}{\left.|x|^{2\left(\frac{1}{2}\right)}|x|^{2\left(\frac{1}{2}\right)} d x\right)^{\frac{1}{2}} \leq C\left\|\frac{d}{d x} a(X, D)\right\|_{\left(L^{2}, L^{2}\right)}\left(\frac{1}{2}\right)^{\frac{1}{2}}|u|_{\Lambda_{0}^{\frac{1}{2}}} .}\right.
\end{aligned}
$$

Finally,

$$
\|\mathrm{a}(\mathrm{X}, \mathrm{D}) \mathrm{f}\|_{\Lambda_{0}^{\frac{1}{2}([0,1], \theta)}} \leq \max \left\{\sup _{\mathrm{x} \in[0,1]}|\mathrm{a}(\mathrm{x}, \cdot)|_{\mathrm{L}^{1}(\mathrm{Z})}, \mathrm{C}\left\|\frac{\mathrm{d}}{\mathrm{dx}} \mathrm{a}(\mathrm{X}, \mathrm{D})\right\|_{\left(\mathrm{L}^{2}, \mathrm{~L}^{2}\right)}\left(\frac{1}{2}\right)^{\frac{1}{2}}\right\}\|\mathrm{f}\|_{\Lambda_{0}^{\frac{1}{2}}([0,1], \theta)}
$$

Theorem 8. Let $0<\varepsilon<1$ and $\mathrm{a}(\mathrm{x}, \xi)$ be $\mathrm{a}$ symbol such that $\left|\Delta_{\xi}^{\alpha} \xi \mathrm{a}(\mathrm{x}, \xi)\right| \leq$ $\mathrm{C}_{\alpha}\langle\xi\rangle^{-\frac{\varepsilon}{2}-(1-\varepsilon)|\alpha|},\left|\partial_{\mathrm{x}}^{\beta} \xi \mathrm{a}(\mathrm{x}, \xi)\right| \leq \mathrm{C}_{\beta}\langle\xi\rangle^{-\frac{\varepsilon}{2}}$, for $0 \leq$ $|\alpha|,|\beta| \leq 1$. If $\frac{1}{2}<s<1$, then $\mathrm{a}(\mathrm{X}, \mathrm{D}): \Lambda_{0}^{\mathrm{s}}([0,1], \theta) \rightarrow \Lambda^{\mathrm{s}}([0,1], \theta)$ is a bounded linear operator.
Proof. If $\frac{1}{2} \leq \mathrm{s}<1$, there exists $2 \leq \mathrm{p}<\infty$ such that $s=1-\frac{1}{\mathrm{p}}$. Applying Theorem 6 to the symbol $i 2 \pi \xi a(x, \xi)$ we obtain $\mathrm{L}^{\mathrm{p}}(0,1)$ - boundedness for the operator $\frac{d}{d x} a(X, D)$. If $u \in \Lambda_{0}^{s}([0,1], \theta)$, then

$$
\begin{aligned}
& \frac{|a(X, D) u(x+h)-a(X, D) u(x)|}{|h|^{s}} \leq C\left|\frac{d}{d x} a(X, D) u\right|_{L^{p}} \leq C\left\|\frac{d}{d x} a(X, D)\right\|_{\left(L^{p}, L^{p}\right)}|u|_{L^{p}} \\
= & C\left\|\frac{d}{d x} a(X, D)\right\|_{\left(L^{p}, L^{p}\right)}\left(\int_{0}^{1} \frac{|u(x)-u(0)|}{|x|^{p^{s}}}|x|^{p^{s}} d x\right)^{\frac{1}{2}} \leq C\left\|\frac{d}{d x} a(X, D)\right\|_{\left(L^{p}, L^{p}\right)}\left(\frac{1}{p}\right)^{\frac{1}{p}}|u|_{\Lambda_{0}^{s}} .
\end{aligned}
$$


Hence,

$$
|\mathrm{a}(\mathrm{X}, \mathrm{D}) \mathrm{u}|_{\Lambda^{\mathrm{s}}} \leq \mathrm{C}\left\|\frac{\mathrm{d}}{\mathrm{dx}} \mathrm{a}(\mathrm{X}, \mathrm{D})\right\|_{\left(\mathrm{L}^{\mathrm{p}, \mathrm{L}^{\mathrm{p}}}\right)}\left(\frac{1}{\mathrm{p}}\right)^{\frac{1}{\mathrm{p}}}|\mathrm{u}|_{\Lambda_{0}^{\mathrm{s}}}
$$

Now, since $|\xi \mathrm{a}(\mathrm{x}, \xi)| \leq \mathrm{C}\langle\xi\rangle^{-\frac{\varepsilon}{2}}$, we get

$$
|\mathrm{a}(\mathrm{x}, \xi)| \leq \mathrm{C}\langle\xi\rangle^{-\frac{\varepsilon}{2}}|\xi|^{-1}, \xi \neq 0 .
$$

Hence, we obtain

$$
M=\sup _{x \in[0,1]}|a(x, \cdot)|_{L^{1}(Z)}<\infty
$$

Therefore

$\leq \begin{gathered}\|\mathrm{a}(\mathrm{X}, \mathrm{D}) \mathrm{f}\|_{\Lambda_{0}^{\mathrm{s}}([0,1], \theta)} \leq \\ \leq \max \left\{\mathrm{M}, \mathrm{C}\left\|\frac{\mathrm{d}}{\mathrm{dx}} \mathrm{p}(\mathrm{X}, \mathrm{D})\right\|_{\left(\mathrm{L}^{\mathrm{p}, \mathrm{L}^{\mathrm{p}}}\right)}\left(\frac{1}{\mathrm{p}}\right)^{\frac{1}{\mathrm{p}}}\right\}\|\mathrm{f}\|_{\Lambda_{0}^{\mathrm{s}}([0,1], \theta)} .\end{gathered}$

Acknowledgement. The authors were supported by the Ministry of Education and Science of the Republic of Kazakhstan, MESRK Grant AP05130994.

\section{References}

1. Agranovich M.S. "Spectral properties of elliptic pseudo-differential operators on a closed curve." Func. Anal. Appl. 13 (1979): 279-281.

2. Agranovich M.S. "On elliptic pseudodifferential operators on a closed curve." Trans. Moscow Math. Soc. 47 (1985): 23-74.

3. Kanguzhin B.E., Tokmagambetov N. and Tulenov K. "Pseudo-differential operators generated by a non-local boundary value problem." Complex
Variables and Elliptic Equations 60, no. 1 (2015): 107-117.

4. Brézis, H. "Analyse fonctionnelle: théorie et applications.” (Paris: Éditions Dunod, 1999), 427.

5. Cardona D. "Holder estimates for pseudodifferential operators on $\mathrm{T}^{1}$." J. Pseudo-Differ. Oper. Appl. 5 (2014): 517-525.

6. Delgado,J. " $\mathrm{L}^{\mathrm{p}}$ bounds for pseudo-differential operators on the torus." Oper. Theory Adv. Appl. 231, (2012): 103-116.

7. Kanguzhin B.E, Sadybekov M.A. "Differential operators on a segment. Distributions of the eigenvalues.” (Almaty: Gylym, 1996), 312.

8. Ruzhansky M, Turunen V. "Pseudodifferential operators and symmetries." (Basel: Birkhauser, 2010), 697.

9. Ruzhansky M, Turunen V. "Quantization of pseudo-differential operators on the torus." $J$. Fourier Anal. Appl. 16 (2010): 943-982.

10. Ruzhansky M, Turunen V. "On the Fourier analysis of operators on the torus. Modern trends in pseudo-differential operators." Operator theory advances and applications 172 (2007): 87-105.

11. Ruzhansky M, Turunen V. "Global quantization of pseudo-differential operators on compact Lie groups, SU(2) and 3-sphere." Int. Math. Res. Not. 11 (2013): 2439-2496.

12. Ruzhansky M, Turunen V. "Sharp garding inequality on compact Lie groups." J. Funct. Anal. 260 (2011): 2881-2901.

13. RuzhanskyM, Wirth J. "On multipliers on compact Lie groups." Funct.Anal.Appl. 47 (2013): 72-75.

14. Taylor M. "Pseudo-differential operators and nonlinear PDE." (Boston (MA): Birkhauser, 1991), 202.

15. Taylor M. "Pseudo-differential operators." (Princeton (NJ): Princeton University Press, 1981), 468. 